\title{
New Type of Charge and Magnetic order in the Ferromagnetic Kondo Lattice
}

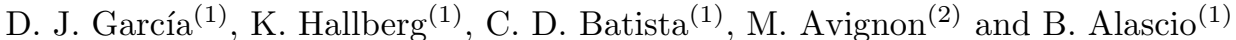 \\ (1)Centro Atómico Bariloche and Instituto Balseiro \\ Comisión Nacional de Energía Atómica, 8400 S.C. de Bariloche, Argentina \\ (2)Laboratoire d'Etudes des Propriétés Electroniques des Solides(LEPES) \\ Centre National de la Recherche Scientifique (CNRS), BP 166, 38042 Grenoble Cedex 9, France
}

(Received April 7, 2018)

\begin{abstract}
We study numerically the one dimensional ferromagnetic Kondo lattice, a model widely used to describe nickel and manganese perovskites. By including a nearest neighbor Coulomb interaction $(\mathrm{V})$ and a superexchange interaction between the localized moments $(\mathrm{K})$, we obtain the phase diagram in parameter space for several dopings at $\mathrm{T}=0$. Due to the competition between double and superexchange, we find a region where the formation of magnetic polarons induces a charge ordered $(\mathrm{CO})$ state which survives also for $\mathrm{V}=0$. This mechanism should be taken into account in theories of charge ordering involving spin degrees of freedom.
\end{abstract}

PACS numbers: 75.10-b, 75.30.Vn, 75.40.Mg

In recent years there has been great interest in the nontrivial interplay of charge, spin and lattice degrees of freedom in strongly correlated electron systems, especially in perovskite transition-metal oxides. One of the most striking phenomena is the simultaneous appearance of charge and spin superstructures. For example, neutron scattering [1] and electron diffraction [2] experiments in $\mathrm{La}_{2-x} \mathrm{Sr}_{x} \mathrm{NiO}_{4}$ showed the presence of charge/lattice and spin modulations with doping-dependent wave vector. Stripe formation together with incommensurate spin fluctuations in High Tc superconductors can also be regarded as a manifestation of similar phenomena [3]. The charge and spin ordering found in many of the doped manganese perovskites also fall in the same category. Experiments have revealed $\mathrm{CO}$ at half filling in $\mathrm{Nd}_{0.5} \mathrm{Sr}_{0.5} \mathrm{MnO}_{3}$ [1] and similar compounds, such as $\operatorname{Pr}_{0.5} \mathrm{Ca}_{0.5} \mathrm{MnO}_{3}$, [5 81. More recent interest has focused on electron doped charge ordered manganites 9 11. $\mathrm{CO}$ has also been found for other dopings as in $\mathrm{Bi}_{1-x} \mathrm{Ca}_{x} \mathrm{MnO}_{3}$ [9] and in $\mathrm{La}_{1-x} \mathrm{Ca}_{x} \mathrm{MnO}_{3}$ (doped with $\mathrm{Pr}$ ) [11] for $x \geqq 0.5$ (few electron region).

The ferromagnetic staircase structure of the CE phase is found in several of the $x=0.5$ manganites and the $\mathrm{CO}$ found in these have been interpreted in terms of orbital ordering [12,13]. The ordering between chains, however, is not yet clear.

Although there have been several attempts to explain the $\mathrm{CO}$ phase theoretically in the half-filled case by considering two $M n$ orbitals 14, 15, 12, with and without intersite Coulomb interaction $V$, and adding strong on-site Coulomb interactions [12], there remains, to the best of our knowledge, no explanation for the existence of $\mathrm{CO}$ in the electron-doped region $(x>0.5)$ [9,10] . Numerical studies for several dopings [16] have included the effect of $V$ and obtained a very rich phase diagram, finding phase separated (for either extreme dopings) and $\mathrm{CO}(x \simeq 0.5)$ regimes. In Ref. [17 the $x=0.5$ two-orbital case is also studied using Monte Carlo techniques, and the $\mathrm{CO}$ phase is stabilized by Jahn-Teller phonons. As mentioned in this work, the $z$-axis stacking of charge and existence of bistripes at $x>0.5$ [10], both penalized by a large Coulomb interaction, indicate that $V$ is smaller than expected and is not enough to understand the CO state.

Unlike CO in non-magnetic materials where the Peierls instability or large intersite Coulomb interactions are required, we will show here that in these magnetic materials charge density waves can result from the formation of magnetic superstructures arising from the presence of competing interactions. By changing the carrier concentration or the relationship between the competing interactions, this new mechanism gives rise to a very rich family of inhomogeneous spin and charge structures.

In order to illustrate this new mechanism we will study here a simplified model where the competing forces are personified by the double and superexchange interactions. The Ferromagnetic Kondo Lattice Model (FKLM), which was devised for manganites, was studied originally by de Gennes [18]. The most intriguing question is what happens in an intermediate regime, where the competing interactions are energetically similar. De Gennes proposed canting of two interpenetrating lattices as the compromise solution of this competition. This concept was also used subsequently in recent analytical approaches [19.20]. Phase separation has also been considered as a possible solution to this competition [21,22].

As will be shown below, we find spin phases which cannot be described in terms of two interpenetrating sublattices nor do they correspond to phase separation.

As a result of the competition between the double exchange (DE) mechanism which delocalizes the hole and the superexchange (SE) between local spins, different phases may appear such as ferromagnetism in one extreme, antiferromagnetism in the other, and dopingdependent modulated charge and spin order in between. For $x=1 / 2$, we find no charge ordering for $V=0$, but away from that concentration we find charge modulation even in the absence of Coulomb interactions. In this case, we find what can be described as an ordered phase of fer- 
romagnetic islands in which carriers are localized. These phases are insulating, but a magnetic field can induce a transition to a metallic phase.

We consider the widely used FKLM. For completeness we add the effect of nearest-neighbors intersite interaction when considering $x=0.5$ :

$$
\begin{aligned}
H= & -t \sum_{i}\left(c_{i \sigma}^{\dagger} c_{i+1 \sigma}+h . c .\right)+U \sum_{i} n_{i \uparrow} n_{i \downarrow}+ \\
& V \sum_{i} n_{i} n_{i+1}+J_{h} \sum_{i} \mathbf{S}_{i} \sigma_{i}+K \sum_{i} \mathbf{S}_{i} \mathbf{S}_{i+1}
\end{aligned}
$$

Here the first term represents the $e_{g}$-electron transfer between nearest-neighbor Mn ions at sites $i$ and $i+1$ (we will take $t$ as the unit of energy), the second and third terms are the on-site and inter-site Coulomb repulsions between these orbitals, $J_{h}$ is the Hund's rule coupling between localized $\mathbf{S}_{i}$, taken to have $S=1 / 2$, and itinerant $\sigma_{i}$ spins and $K$ is the superexchange between local spins.

We will fix the values $J_{h}=20$ and $U=10$, so that the only free parameters are $V$ and $K$. For the large $J_{h}$ used in this paper the results are not sensitive to $U$ since double occupancy is supressed by $J_{h}$. We consider here the case of one non-degenerated $e_{g}$ orbital, and defer the analysis of the role of orbital ordering for future study. The ground state of this model has also been calculated by other authors [16] using the finite-system DMRG 23,24. Here we have taken special care in the growing procedure in order not to frustrate the system in the cases when charge and spin order are expected [25]. With this consideration, very accurate results were obtained with a discarded weight lower than $10^{-4}$ for the largest systems presented here.

Let us consider first the case $V=0$, in order to isolate the two competing interactions $K$ and $t$. For any finite concentration there is a fully polarized ferromagnetic $(\mathrm{F})$ phase below some critical value of $K / t$, and an antiferromagnetic (AF) phase for sufficiently large $K / t$. This can be seen in Fig. 1 for $x=0.5$. In the intermediate regime we get a phase $(0.2<K / t<0.4)$ with clear peaks in the spin structure factor $S(q)$ at $q= \pm \pi / 2$ (see Fig. 1a) . These peaks increase logarithmically with $L$ (see Fig. 2a) indicating a power law decay of the spin-spin correlation function in real space. This power law decay is a consequence of the fact that no long range order can be sustained in a one dimensional model with $S U(2)$ symmetry. This structure has been obtained with quantum Montecarlo using classical spins and interpreted as a spiral state with pitch $q=\pi / 2$ [26]. Instead our results on the real space spin-spin correlation function show ferromagnetic pairs coupled antiferromagnetically:( $\uparrow \downarrow \downarrow \uparrow \uparrow \downarrow \downarrow \ldots)$. Our classical Montecarlo calculations 27] confirm this picture.

Now if we look at the charge, we find that even though there is no charge ordering in any of these phases, the charge-charge correlation functions shown in Fig 1b are essentially different. While the ferromagnetic case $(K / t=0.1)$ corresponds to a spinless metallic phase (the peak at $q=2 k_{F}=\pi$ is a consequence of Friedel oscillations), in the $\mathrm{AF}$ case $(K / t=1) N(q=\pi)$ the Friedel oscillations loose weight (see Fig. 1). Concerning the $q=\pi / 2$ phase, it can be seen from Fig. 1b and Fig. $2 \mathrm{~b}$ that there is no charge ordering for this phase, even when the charge-charge correlation function is quite different compared to the ferromagnetic case. This change can be understood if we consider that the charges are 'localized' in bonds due to the spin structure $(\uparrow \uparrow \downarrow \downarrow \uparrow \uparrow \downarrow \downarrow \ldots)$, which would give an insulating character to this phase (preliminar calculations of the Drude weight seem to confirm this view [28]). Each charge induces a ferromagnetic island of two spins and tends to be localized in the bond in order to gain kinetic energy. The shape of $N(q)$ is closer to $(1-\cos q) / 4$ as a consequence of the enhancement of the charge correlations within an island. From this point of view it is easy to understand the absence of charge ordering, because both sites are completely equivalent in each island. However, this would not be the case if the tendency to form ferromagnetic islands surrounding each charge were preserved for lower electron concentrations. If we consider, for example, $x=2 / 3$ the expected spin phase may be represented schematically as $\uparrow \uparrow \uparrow \downarrow \downarrow \downarrow \uparrow \uparrow \uparrow \downarrow \downarrow \downarrow \ldots$, where the spins cluster in the form of magnetic polarons [29,30]. In this case, the centers of each island are not equivalent to the borders, and the charge will tend to accumulate in the middle of the polarons. For this reason we expect to get $\mathrm{CO}$ together with a spin density wave for electron concentrations lower than 0.5 . This scheme is confirmed by Fig. 3, where we show the charge and spin structure factors calculated for $x=2 / 3$ and $K / t=0.25 . S(q)$ clearly shows a peak at $q=\pi / 3$, while $N(q)$ has a pronounced peak at $q=2 \pi / 3$ (the peak at small $q$ in $N(q)$ for the $x=1 / 3$ case is due to a kink in the center of the open chain). Fig.2 shows that the intensity of these peaks scale logarithmically with $L$ for $S(q=\pi / 3)$ and linearly for $N(q=2 \pi / 3)$ which is a clear evidence of long-range charge ordering. The same calculations for $x=0.2,0.25$ show spin and charge correlation functions consistent with the formation of 4 - and 5 -sites spin islands containing one electron each. These structures could be regarded as a crystallization of the magnetic polarons described in references 29,30] for the dilute limit. It is interesting to note that $\mathrm{CO}$ is induced by spin ordering and vice versa, demostrating that the formation of these superstructures is a consequence of the interplay between charge and spin degrees of freedom.

In the case $x=1 / 3$, it is evident from Figs. 2 and 3 that there is also a spin and charge-ordered state for this concentration. Both $N(q)$ and $S(q)$ now have peaks at $q=2 \pi / 3$. This result can be understood clearly with the following image for the magnetic structure: $\uparrow \uparrow \downarrow \uparrow \uparrow \downarrow \uparrow \uparrow \uparrow \downarrow$, while the charge is distributed with one electron in each pair of up spin sites, and one in each down spin site. 
The validity of the image described above can be easily tested using a classical approach. We calculate the contributions of double exchange and superexchange to the energy of the different possible phases for each value of the concentration, assuming that the hopping vanishes at the antiferromagnetic bonds. For example in the case $x=1 / 2$, we obtain $-2 t / \pi+K$ for the ferromagnetic phase, $-t / 2$ for the $q=\pi / 2(\uparrow \uparrow \downarrow \downarrow \uparrow \uparrow \downarrow \downarrow)$ phase, and $-K$ for the antiferromagnetic phase. The sequence of stable phases when increasing $K / t, \mathrm{~F} \rightarrow \pi / 2 \rightarrow \mathrm{AF}$ is obtained in agreement with the numerical results. In this classical picture a canted AF phase has a slightly lower energy than the pure AF phase, but the $\pi / 2$ phase remains stable over a certain region in $K / t$. The same procedure leads to similar conclusions at $x=1 / 3$ and $2 / 3$.

It is also of interest to consider the effect of an intersite Coulomb interaction $V$ at $x=1 / 2$, because at this concentration it is most effective in inducing charge ordering. All phases exhibit charge ordering above a critical value of $V$. In Fig.4 we show a tentative phase diagram in $V$ and $K$. Our calculations for different values of $K$ indicate a first-order transition from the fully polarized ferromagnetic phase to the one with $q=\pi / 2$ spin order. This metal-insulator transition can be induced by the application of a magnetic field. As we mentioned above this insulator to metal transition can also be obtained by applying a magnetic field that polarizes the spins, thus delocalizing the electrons due to the double exchange interaction. At higher $K / t$ the latter evolves into the $\mathrm{AF}$ phase.

The Coulomb repulsion $V$ inhibits the double-exchange mechanism by reducing the mobility of the carriers. This reduces the phase space for the ferromagnetic order. As $K$ increases, the critical value of $V$ for charge ordering diminishes from the spinless value $2 t$ valid for the saturated ferromagnetic regime. For $V>>t$ we obtain the

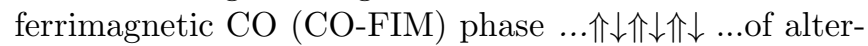
nating $S=1 / 2$ and $S=1$ spins.

In summary, we have presented numerical evidence of the existence of a new type of simultaneous charge and spin ordering in the FKLM. This mechanism, induced by the competition between $\mathrm{DE}$ and $\mathrm{SE}$, is based on a striking interplay between charge and spin degrees of freedom. As we have shown in previous works [29],30], in the dilute limit each carrier polarizes its surroundings forming ferromagnetic polarons. The size of these polarons is governed by the ratio $K / t$. We could interpret our results as an indication that if this size is similar to the mean separation between carriers, then $\mathrm{SE}$ tends to order these islands separating them by antiferromagnetic interphases. Of course, this is an approximate image because there are quantum magnetic fluctuations between and inside the islands. While previous work reported the need of coulomb interaction $V$ 16 or electron-phonon coupling $\lambda[?]$ to stabilize a charge-ordered phase, we show that it arises naturally considering the interplay between charge and spin degrees of freedom.

A great variety of experiments have found the presence of charge and spin ordering in manganites. The extraordinary colossal magnetoresistance effect for $\mathrm{La}_{0.5} \mathrm{Ca}_{0.5} \mathrm{MnO}_{3}$ involves the abrupt destabilization of a $\mathrm{CO}-\mathrm{AF}$ state by a magnetic field [31]. Insulating chargeordered and metallic ferromagnetic regions coexist in $\left(\mathrm{La}_{0.5} \mathrm{Nd}_{0.5}\right)_{2 / 3} \mathrm{Ca}_{1 / 3} \mathrm{MnO}_{3}$ [32] and $\operatorname{Pr}_{0.7} \mathrm{Ca}_{0.3} \mathrm{MnO}_{3}$ [33, 34]. Both phenomena indicate that the $\mathrm{CO}$ phase is very close in energy to the FM state. In addition, the z-axis stacking of charge and existence of bistripes at $x>1 / 2$ [10], indicate that $\mathrm{CO}$ is not driven by a Coulomb repulsion. These observations are consistent with a picture where spin and charge ordering are driven by the strong interplay between charge and spin degrees of freedom. For these reasons, the phenomenon presented here could be the underlying physical mechanism for the stabilization of spin and charge structures in manganites.

Our model calculations fail to include several effects that may play important roles in real systems like JahnTeller distortions and orbital degeneracy. However it is important to understand clearly that charge ordering can be induced simply as a result of the competition between $\mathrm{DE}$ and SE as shown here. Considerable work remains to be done. For example, it would be desirable to complete a $K / t$ vs. $n$ phase diagram.

We thank Silvia Bacci for helping us with computer programs and B. Normand and D. Poilblanc for useful sugestions and discussions. Three of us (D. G., C.D.B. and K. H.) are supported by the Consejo Nacional de Investigaciones Científicas y Técnicas (CONICET). B. A. is partially supported by CONICET. We would like to acknowledge support from the Program for scientific collaboration between France and Argentina ECOS-SECyT A97E05 and the University of Buenos Aires where part of this work has been done.

[1] S. Hayden et al. Phys. Rev. Lett. 68, 1061 (1992).

[2] C. Chen et al., Phys. Rev. Lett. 71, 2461 (1993).

[3] J. M. Tranquada, B. J. Sternlieb, J. D. Axe, Y. Nakamura, S. Uchida, Nature 375, 561 (1995).

[4] H. Kawano et al., Phys. Rev. Lett 78, 4253 (1997).

[5] Y. Tomioka et al., Phys. Rev. B 53, R1689 (1996).

[6] J. Li et al., Phys. Rev. B 57, R3205 (1998).

[7] Y. Moritomo et al., 56, 14879 (1997).

[8] C. Chen et al., Phys, Rev. Lett. 76, 4042 (1996).

[9] H. Liu et al., Phys. Rev. Lett. 81, 4684 (1998); W. Bao et al., Phys. Rev. Lett. 78, 543 (1997).

[10] S. Mori et al., Nature 392, 473 (1998).

[11] M. Uehara et al., Nature 399, 560 (1999); Z. Jirac et al., J. M. M. Mat. 53, 153 (1985).

[12] J. Van den Brink et al., cond/matt 9910253. 
[13] T. Hotta et al., cond/matt 9911248

[14] T. Mizokawa et al., Phys. Rev. B 56, R493 (1997)

[15] G. Jackeli et al., cond-mat/9910391

[16] A. Malvezzi et al., Phys. Rev. B 59, 7033 (1999).

[17] S. Yunoki, A. Moreo, and E. Dagotto, Phys. Rev. Lett. 81, 5612 (1998).

[18] P-G. de Gennes, Phys. Rev. 118, 141 (1960).

[19] H. Aliaga et al, Solid State Comm. 110, 525 (1999).

[20] D. P. Arovas, F. Guinea, Phys. Rev. B 58, 9150 (1998).

[21] M. Yu Kagan et al, cond-mat 9804213

[22] A. Moreo, S. Yunoki, and E.Dagotto, Science 283, 2034 (1999).

[23] S. White, Phys. Rev. Lett. 69, 2863 (1992); Phys. Rev. B 48, 10345 (1993).

[24] Density Matrix Renormalization, Lectures Notes in Physics, Edited by I. Peschel, X. Wang, M. Kaulke, and K. Hallberg, Springer Verlag (1999).

[25] D. García et al, in preparation.

[26] S. Yunoki and A. Moreo, Phys. Rev. B 58, 6403 (1998).

[27] H. Aliaga et al, in preparation.

[28] S. Capponi and D. Poilblanc, private communicaton.

[29] C. D. Batista, J. Eroles, M. Avignon, B. Alascio, Phys. Rev. B 58, R14689 (1998).

[30] C. D. Batista, J. Eroles, M. Avignon, B. Alascio, preprint.

[31] Y. Tomioka et al, J. Phys. Soc. Jpn. 64, 3626 (1995); Phys. Rev. B 53, R1689 (1996); H. Yoshizawa et al, Phys. Rev. B 52, R13145 (1995).

[32] M. R. Ibarra et al, Phys. Rev. B 57, 7446 (1998).

[33] D. E. Cox et al, Phys. Rev. B 57, 3305 (1998).

[34] V. Kiryukhin et al, Nature (London) 386, 813 (1997).

Figure 1: Spin and charge structure factors for $x=0.5$ and $L=28$.

Figure 2: Size dependence of the peaks of the spin and charge structure factors.

Figure 3: Spin and charge structure factorsfor $x=$ $1 / 3,2 / 3$ and $L=30$.

Figure 4: Phase diagram $\mathrm{K} / \mathrm{t}$ vs. $\mathrm{V} / \mathrm{t}$ for $x=0.5$. The circles indicate the points where calculations have been performed. 

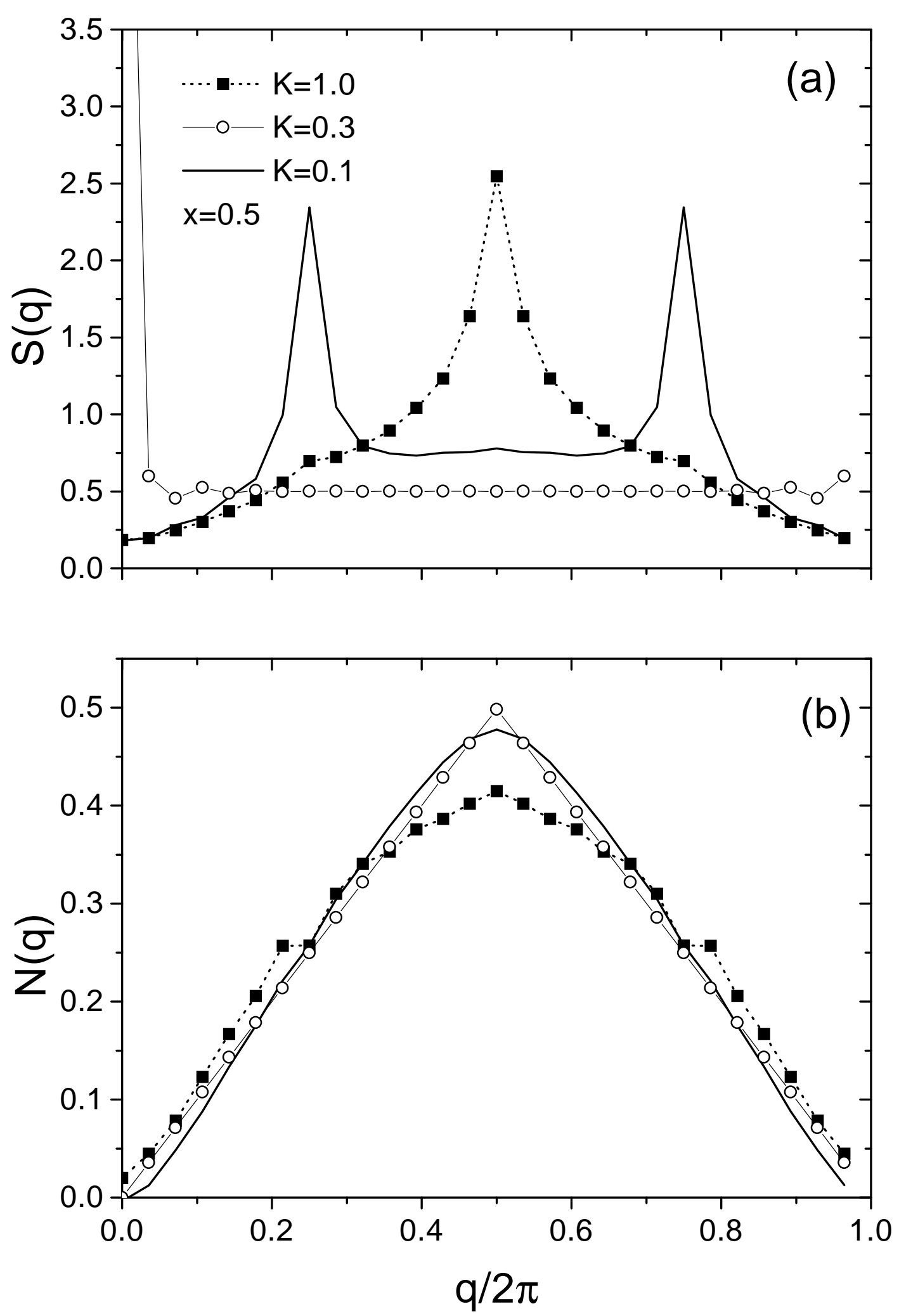

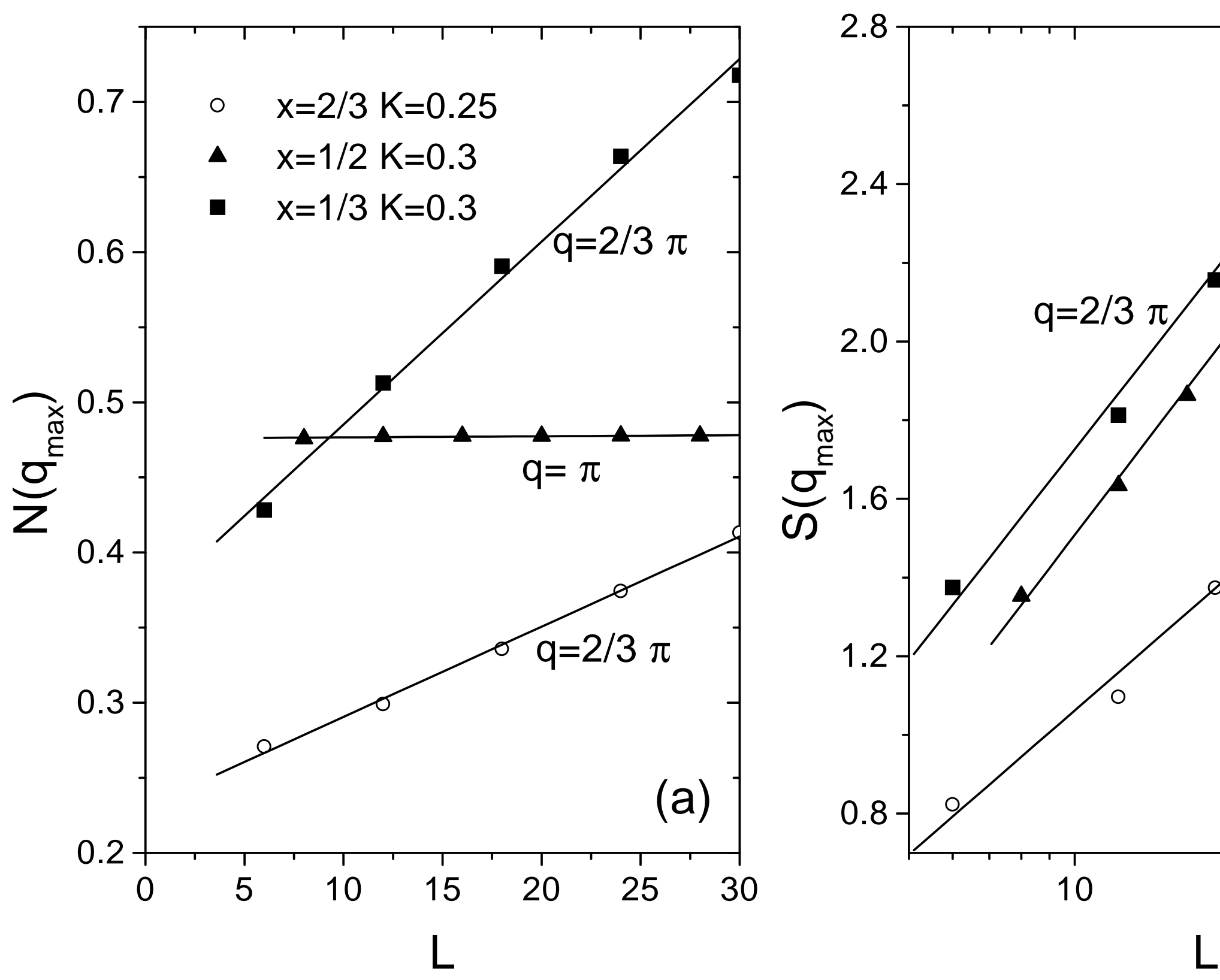
Figure 3
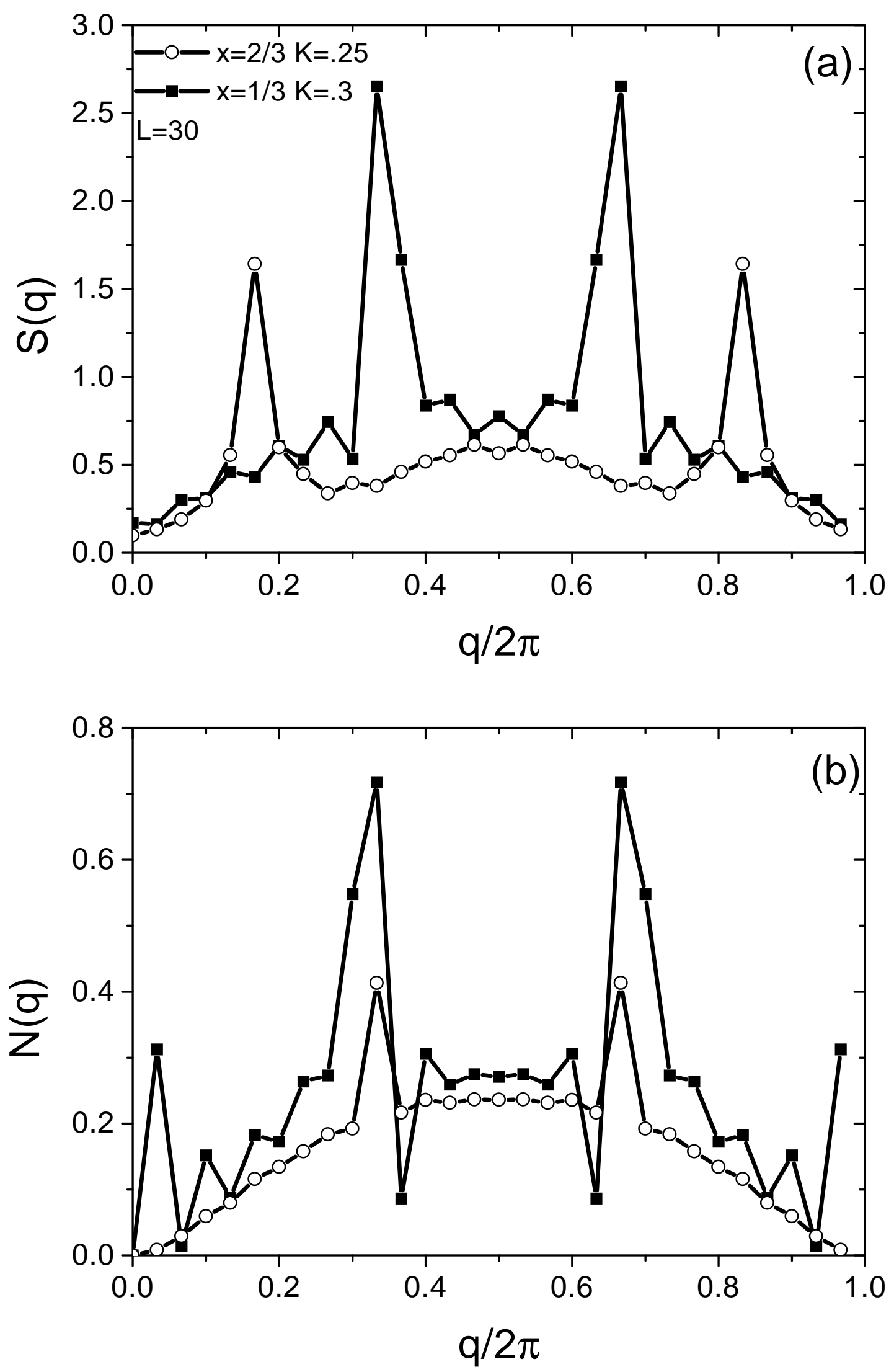
Figure 4

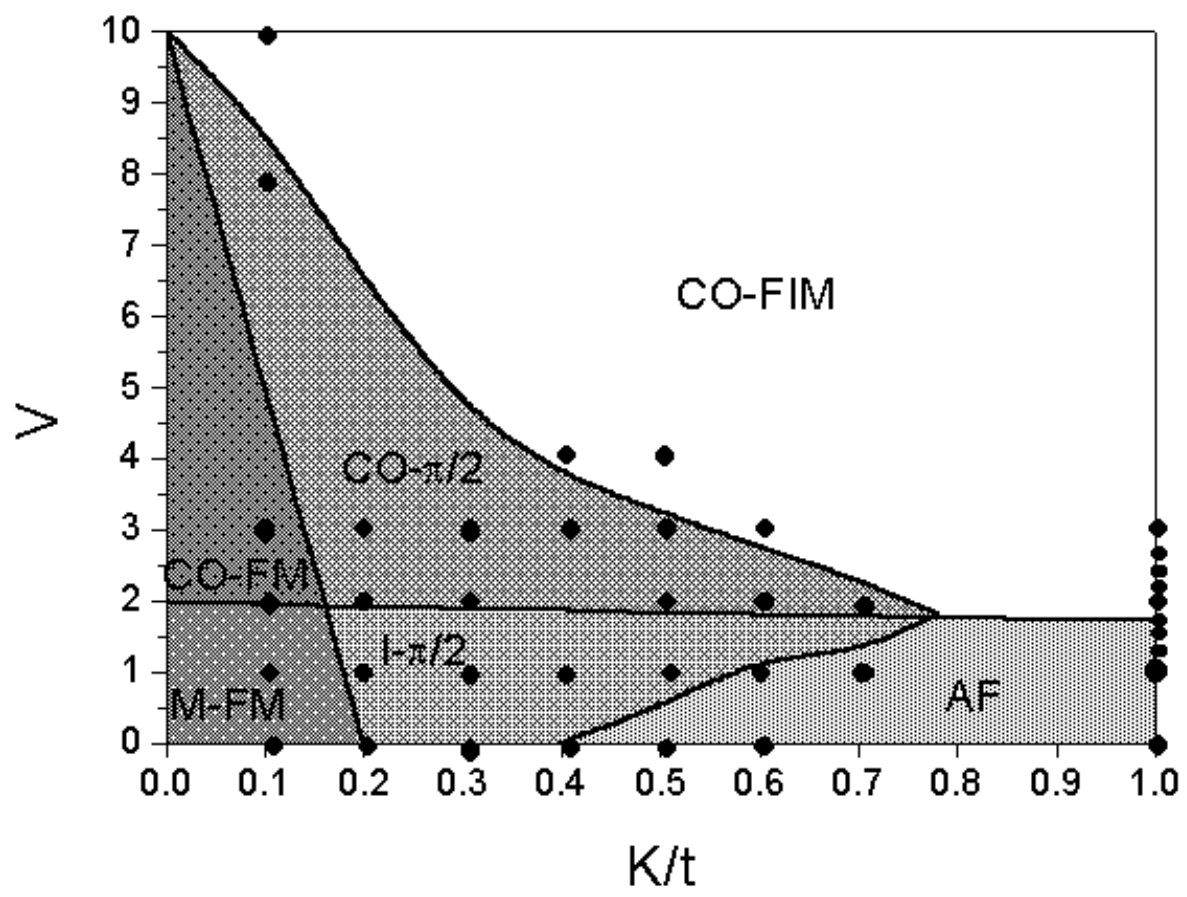

